Pacific Journal of Mathematic 


\title{
A NOTE ON THE LÖWNER DIFFERENTIAL EQUATIONS
}

\author{
MAURICE HeINS \\ Charles Löewner in Memoriam
}

\begin{abstract}
The object of the present note is to indicate a derivation of the Löwner differential equations [1] based on the derivation of an associated differential equation for Green's function of the variable region relative to the defining parameter. Decisive in our treatment is the use of a certain normalized minimal positive harmonic function on the variable region. In fact, our starting point was the feeling that the Poisson kernel asserted its presence so strongly in the Löwner differential equations that the concomitant presence of a normalized minimal positive harmonic function on the variable region should appear naturally in the study of the question. We shall see that this is the case. A technical advantage of the present approach is that the "tip" lemmas of the classical proof are dispensed with.
\end{abstract}

It would be of interest to see whether the indicated method, which is available for other families of harmonic functions monotone justifying in a parameter, has useful applications to the theory of harmonic functions.

2. Let $\gamma$ be a Jordan arc with parametric domain $[0, T]$ such that $0<|\gamma(t)|<1$ for $0 \leqq t<T$ and $|\gamma(T)|=1$. Let $A_{t}$ denote the complement of the set $\gamma(\{t \leqq s<T\})$ with respect to the open unit disk, $0 \leqq t \leqq T$. Let $g_{t}$ denote Green's function for $A_{t}$ with pole at 0 . The continuous dependence of $g_{t}$ on the parameter $t$ is an elementary matter (minimal property of Green's function, the PhragménLindelöf boundary maximum principle). We let $\alpha(t)$ denote $\lim _{z \rightarrow 0}$ $\left[g_{t}(z)+\log |z|\right]$. We note that $\alpha: t \rightarrow \alpha(t)$ is an increasing continuous function which satisfies $\alpha(T)=0$. We reparametrize $\gamma$, as in the original Löwner argument, by composing $\gamma$ with

$$
t \longrightarrow \operatorname{inv} \alpha[t+\alpha(0)], 0 \leqq t \leqq-\alpha(0),
$$

so that for the new $\gamma$ we have $T=-\alpha(0)$ and $\alpha(t)=\alpha(0)+t$. [The notation "inv" is used to denote the inverse of a univalent function.] We let $G$ be defined by

$$
G(z, t)=g_{t}(z), z \in A_{t}, 0 \leqq t \leqq T .
$$


Given a function $F$ having as domain a subset of $C \times R$, we denote by $D_{2} F(a, b)$ the derivative of $t \rightarrow F(a, t)$ at $b$ and by $D_{1} F(a, b)$ and $\bar{D}_{1} F(a, b)$ the complex differential coefficients of $z \rightarrow F(z, b)$ at $a$, the obvious conventions holding. Our first step is to establish the existence of $D_{2} G$ and to obtain information about it. We remark that the logarithmic singularity of $g_{t}$ at 0 is harmless. The difference quotient

$$
\frac{G(z, t)-G(z, s)}{t-s}, 0 \leqq s<t \leqq T
$$

defines a positive harmonic function on $A_{s}$ which takes the value 1 at 0 and vanishes continuously at each point of the frontier of $A_{t}$.

To control the limiting behavior of (1) as $(s, t) \rightarrow(\sigma, \sigma), 0 \leqq \sigma \leqq$ $T$, we make use of the boundary behavior of the Riemann mapping function for a simply-connected Jordan region and the following standard lemma of Harnack type.

Lemma. Let $m(z)=(1-|z|) \quad(1+|z|)^{-3}$ and $M(z)=(1+|z|)$ $\times(1-|z|)^{-3}$. Let $a$ and $b$ be points of the semi-circular disk $\{\operatorname{Im} z>$ $0,|z|<1\}$. Let $u$ be nonnegative and harmonic on this set and vanish continuously on the diameter. Then

$$
[M(b)]^{-1} \frac{u(b)}{\operatorname{Im} b} \leqq[m(a)]^{-1} \frac{u(a)}{\operatorname{Im} a} .
$$

[A proof of this lemma is readily given with the aid of Schwarzian reflexion and the Poisson integral for a circular disk.]

Suppose that $\left(s_{n}, t_{n}\right) \rightarrow(\sigma, \sigma)$, where $0 \leqq s_{n}<t_{n} \leqq 1$. Then some subsequence of the sequence of difference quotients (1), given by $s=s_{n}$ and $t=t_{n}$, converges, uniformly on compact subsets of $A_{\sigma}$, to a positive harmonic function on $A_{\sigma}$ which takes the value 1 at 0 . Using the boundary behavior of the Riemann mapping function when a Jordan boundary lies at hand and the stated lemma, we see that the limit function in question vanishes continuously at each point of the frontier of $A_{\sigma}$, the "tip" $\gamma(\sigma)$ excepted.

We introduce the normalized Riemann mapping function $f_{t}$, mapping the open unit disk onto $A_{t}$ and satisfying $f_{t}(0)=0, f_{t}^{\prime}(0)>$ 0 . From the continuity of $t \rightarrow \mathrm{g}_{t}$, we infer the continuity of $t \rightarrow \operatorname{inv} f_{t}$ and thence the continuity of $t \rightarrow f_{t}$. Of course, the term "continuity" is to be construed in the sense of uniform limits on compact subsets. We let $\kappa(t)$ denote the unique preimage of $\gamma(t)$ with respect to the continuous extension of $f_{t}$ to the closed unit disk. If $h$ is a positive harmonic function on $A_{t}$ taking the value 1 at 0 and vanish- 
ing continuously at each point of the frontier of $A_{t}$, the "tip" $\gamma(\mathrm{t})$ excepted, then

$$
h\left[f_{t}(z)\right]=\operatorname{Re}\left[\frac{\kappa(t)+z}{\kappa(t)-z}\right],|z|<1 .
$$

It follows that there is at most one $h$ having the stated property. Using (2) as a defining condition for $h$, we see that such $h$ exist. We denote the unique $h$ in question (which is a normalized minimal positive harmonic function on $A_{t}$ ) by $h_{t}$.

Combining the results of the preceding two paragraphs we conclude that the difference quotient (1) tends to $h_{\sigma}$ as $(s, t) \rightarrow(\sigma, \sigma)$ and that, in fact, the uniformity of the limit process holds on compact subsets of $A_{\sigma}$. We let $H$ be defined by

$$
H(z, t)=h_{t}(z), z \in A_{t}, 0 \leqq t \leqq T .
$$

We see that the following differential equation, which will serve as a basis for the derivation of the Löwner differential equations, holds:

$$
D_{2} G=H \text {. }
$$

Continuity of $t \rightarrow h_{t}$ and $\kappa: t \rightarrow \kappa(t)$. A second application of the boundary behavior of the Riemann mapping function for Jordan regions and the lemma yields the continuity of $t \rightarrow h_{t}, 0 \leqq t \leqq T$. It suffices to establish the fact that if $t_{n} \rightarrow \sigma$, then some subsequence of $\left(h_{t_{n}}\right)$ tends to $h_{\sigma}$. Using the continuity of $t \rightarrow h_{t}$, the continuity of $t \rightarrow f_{t}$, and (2) we shall now conclude the continuity of $\kappa$. Indeed, if $t_{n} \rightarrow \sigma$ and $\kappa\left(t_{n}\right) \rightarrow \alpha$, we obtain, using (2), the equality

$$
h_{\sigma}\left[f_{\sigma}(z)\right]=\operatorname{Re}\left(\frac{\alpha+z}{\alpha-z}\right),|z|<1,
$$

and hence $\alpha=\kappa(\sigma)$. The continuity of $\kappa$ follows.

3. The Löwner differential equations. The equations bear on the functions $F, \Psi$, and $\Theta$, which will now be introduced.

$F$. We define $F$ by $F(z, t)=f_{t}(z),|z|<1,0 \leqq t \leqq T$. It is convenient to have available $\Phi$ defined by $\Phi(z, t)=\operatorname{inv} f_{t}(z), z \in A_{t}$, $0 \leqq t \leqq T$. Its role is auxiliary. The function $\Phi$ is useful as a link between $F$ and $G=-\log |\Phi|$.

$\Psi$. The function $\Psi$ is defined by $\Psi(z, t)=$

inv $f_{t}\left[f_{0}(z)\right],|z|<1,0 \leqq t \leqq T$. This is the first function studied by Löwner in his classical paper. There is an identity involving $\Phi$ and $\Psi$ :

$$
\Psi(z, t)=\Phi\left[f_{0}(z), t\right] .
$$


$\Theta$. The function $\Theta$ is specified by the requirement that, $z \rightarrow$ $\Theta(z, t)$ is the inverse of $z \rightarrow \Psi(z, t),|z|<1,0 \leqq t \leqq T$. From

$$
z=\Psi[\Theta(z, t), t]=\operatorname{inv} f_{t}\left\{f_{0}[\Theta(z, t)]\right\}
$$

for $(z, t)$ in the domain of $\Theta$, we obtain for such $(z, t)$ the identity

$$
F(z, t)=f_{t}(z)=f_{0}[\Theta(z, t)] .
$$

The equation (3) yields a corresponding equation for $\Phi$. Indeed, let $\tilde{h}_{t}$ denote the analytic function with domain $A_{t}$ satisfying $\widetilde{h}_{t}(0)=$ 1 , Re $\widetilde{h}_{t}=h_{t}$, and let $\widetilde{H}$ be defined by $\widetilde{H}(z, t)=\widetilde{h}_{t}(z), z \in A_{t}, 0 \leqq t \leqq$ $T$. Clearly, the function $\widetilde{H}$ is continuous on its domain. To derive an equation bearing on $\Phi$, we introduce $G_{1}$ having the same domain as $G$ which satisfies $G_{1}(z, t)=G(z, t)+\log |z|, z \neq 0, G_{1}(0, t)=\alpha(0)+$ $t$, and thereupon $\widetilde{G}_{1}$ with the same domain and satisfying the condition that $z \rightarrow \widetilde{G}_{1}(z, t)$ is the analytic function with real part $z \rightarrow G_{1}(z$, $t$ ) satisfying $\widetilde{G}_{1}(0, t)=G_{1}(0, t)$. It is readily verified that

$$
D_{2} G_{1}=H,
$$

the limit process being uniform in the sense indicated above. It follows, in view of the normalization made on $\widetilde{G}_{1}$, that

$$
D_{2} \widetilde{G}_{1}=\widetilde{H} .
$$

Using the relation

$$
\Phi(z, t)=z \exp \left[-\widetilde{G}_{1}(z, t)\right],
$$

we are led to the equation

$$
D_{2} \Phi=-\widetilde{H} \Phi .
$$

From (4) and (6) we obtain

$$
\begin{aligned}
D_{2} \Psi(z, t) & =-\widetilde{H}\left[f_{0}(z), t\right] \Psi(z, t) \\
& =-\widetilde{h}_{t}\left[f_{0}(z)\right] \Psi(z, t) \\
& =-\left[\left(\widetilde{h}_{t} \circ f_{t}\right) \circ\left(\operatorname{inv} f_{t} \circ f_{0}\right)(z)\right] \Psi(z, t),
\end{aligned}
$$

and, consequently, the Löwner equation

$$
D_{2} \Psi(z, t)=-\frac{\kappa(t)+\Psi(z, t)}{\kappa(t)-\Psi(z, t)} \Psi(z, t),
$$

$(z, t)$ in the domain of $\Psi$.

The equation for $F$. From (6) and the continuity of $\tilde{H}, \Phi, D_{1} \Phi$, $\widetilde{D}_{1} \Phi$ (trivially, since it vanishes), we conclude that $\Phi$ has the $C^{\prime}$ property and so is differentiable. Since $F$ is continuous on its domain, $D_{1} \Phi$ is nowhere zero, and the identity, 


$$
\Phi[F(z, t), t]=z,
$$

$|z|<1,0 \leqq t \leqq T$, prevails, it is a standard matter of the differential calculus to conclude the existence of $D_{2} F$ and the identity,

$$
\begin{gathered}
D_{1} \Phi[F(z, t), t] D_{2} F(z, t)+D_{2} \Phi[F(z, t), t]=0, \\
|z|<1,0 \leqq t \leqq T .
\end{gathered}
$$

It is elementary that

$$
D_{1} \Phi[F(z, t), t] D_{1} F(z, t)=1,
$$

for the same $(z, t)$. From (8) and (6) we obtain

$$
D_{1} \Phi[F(z, t), t] D_{2} F(z, t)=z \frac{\kappa(t)+z}{\kappa(t)-z},
$$

and thereupon using (9) the equation

$$
D_{2} F(z, t)=D_{1} F(z, t) z \frac{\kappa(t)+z}{\kappa(t)-z},
$$

$|z|<1,0 \leqq t \leqq T$.

That $\Theta$ satisfies the equation (10), $\Theta$ replacing $F$, on its domain, is immediate from (10), and the identities obtained from (5) by differentiation. The $C^{\prime}$ property of $F$ follows from the continuity of $D_{1} \Phi$, $D_{2} \Phi$ and $F$ on their respective domains and the identities (8) and (9) as well as the non-vanishing of $D_{1} \Phi$. The $C^{\prime}$ property $\Theta$ is now concluded with the aid of (5).

\section{REFERENCE}

1. K. Löwner, Untersuchungen über schlichte konforme Abbildungen des Einheitskreises I, Math. Ann., 89 (1923), 103-121.

Received December 7, 1970.

UNIVERSITY OF ILLINOIS 



\section{PACIFIC JOURNAL OF MATHEMATICS}

\section{EDITORS}

H. SAMELSON

Stanford University

Stanford, California 94305

C. R. HobBY

University of Washington

Seattle, Washington 98105
J. DugundjI

Department of Mathematics

University of Southern California

Los Angeles, California 90007

RICHARD ARENS

University of California

Los Angeles, California 90024

\section{ASSOCIATE EDITORS}

E. F. BECKENBACH

B. H. NeUMaNN

F. WOLF

K. YoshidA

\section{SUPPORTING INSTITUTIONS}

UNIVERSITY OF BRITISH COLUMBIA

CALIFORNIA INSTITUTE OF TECHNOLOGY

UNIVERSITY OF CALIFORNIA

MONTANA STATE UNIVERSITY

UNIVERSITY OF NEVADA

NEW MEXICO STATE UNIVERSITY

OREGON STATE UNIVERSITY

UNIVERSITY OF OREGON

OSAKA UNIVERSITY
UNIVERSITY OF SOUTHERN CALIFORNIA STANFORD UNIVERSITY

UNIVERSITY OF TOKYO

UNIVERSITY OF UTAH

WASHINGTON STATE UNIVERSITY UNIVERSITY OF WASHINGTON

$\stackrel{*}{*} \stackrel{*}{*} \stackrel{*}{*}$ AMERICAN MATHEMATICAL SOCIETY
NAVAL WEAPONS CENTER 


\section{Pacific Journal of Mathematics}

Vol. 39 , No. 1

May, 1971

Charles A. Akemann, A Gelfand representation theory for $C^{*}$-algebras ....

Sorrell Berman, Spectral theory for a first-order symmetric system of

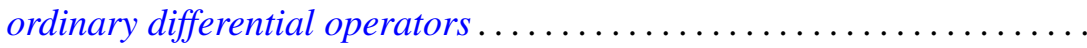

Robert L. Bernhardt, III, On splitting in hereditary torsion theories ........

J. L. Brenner, Geršgorin theorems, regularity theorems, and bounds for determinants of partitioned matrices. II. Some determinantal identities ..........................................

Robert Morgan Brooks, On representing $F^{*}$-algebras .............. 51

Lawrence Gerald Brown, Extensions of topological groups........... 71

Arnold Barry Calica, Reversible homeomorphisms of the real line ........ 79

J. T. Chambers and Shinnosuke Oharu, Semi-groups of local Lipschitzians in

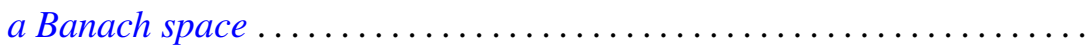

Thomas J. Cheatham, Finite dimensional torsion free rings .............

Byron C. Drachman and David Paul Kraines, A duality between

transpotence elements and Massey products ...................

Richard D. Duncan, Integral representation of excessive functions of a

Markov process ......................................

George A. Elliott, An extension of some results of Takesaki in the reduction

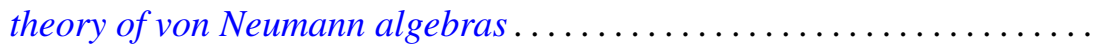

Peter C. Fishburn and Joel Spencer, Directed graphs as unions of partial

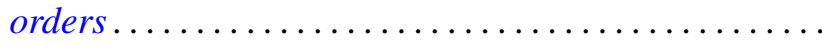

Howard Edwin Gorman, Zero divisors in differential rings ...

Maurice Heins, A note on the Löwner differential equations...

Louis Melvin Herman, Semi-orthogonality in Rickart rings. .

David Jacobson and Kenneth S. Williams, On the solution of linear G.C.D.

equations

Michael Joseph Kallaher, On rank 3 projective planes ... . .

Donald Paul Minassian, On solvable $O^{*}$-groups ...........

Nils Øvrelid, Generators of the maximal ideals of $A(\bar{D})$

Mohan S. Putcha and Julian Weissglass, A semilattice decomposition into

semigroups having at most one idempotent ............

Robert Raphael, Rings of quotients and $\pi$-regularity ....

J. A. Siddiqi, Infinite matrices summing every almost periodic sequence. .

Raymond Earl Smithson, Uniform convergence for multifunctions ...

Thomas Paul Whaley, Mulitplicity type and congruence relations in

universal algebras...

Roger Allen Wiegand, Globalization theorems for locally finitely generated modules... 